\title{
On the uniqueness in Cauchy's problem for elliptic equations
}

By

\author{
Kazunari HAYASHIDA
}

1. It is well known that the uniqueness in Cauchy problem holds for the second order elliptic equation ([1], [4], etc.). On the other hand Mergelyan [9] showed in 1956 that the harmonic function vanishes identically if the Cauthy data tends rapidly to zero at a point along the initial surface. This fact has been extended immediately for the second order elliptic epuation by Landis [7] and Lavrentév [8]. In this note we shall try to extend their results to more general elliptic equations.

Let $\Gamma$ be a smooth Cauchy initial surface in a $N$ dimensional space $R^{N}$ and $\Omega$ be a domain such that the boundary of $\Omega$ contains $\Gamma$. And we consider an elliptic operator $L$ of order $m$ defined in $\bar{\Omega}$. Then we shall say that the strong Cauchy uniqueness property holds for $L$ and $\Gamma$, if any solution $u$ satisfying two following conditions vanishes identically in $\Omega$ :

(i) $u \in C^{m}(\Omega)$ and $L u=0$ in $\Omega$.

(ii) At a point $P_{0}$ in the interior of $\Gamma$, the Cauchy data of $u$ tends to zero along $\Gamma$ in such a way that

$$
\begin{aligned}
\frac{\partial^{|\alpha|} u(P)}{\partial x_{1}^{\alpha_{1}} \cdots \partial x_{n}^{\alpha_{n}}}= & o\left(\exp \left(-\left|P-P_{0}\right|^{-\delta}\right)\right) \\
& \left(P \rightarrow P_{0}, P \in \Gamma\right) \quad|\alpha| \leqq m-1,
\end{aligned}
$$

where $\delta$ is a positive number depending only on $L$ and $\Gamma$.

First we consider the case where $L$ is of second order $(m=1)$

$$
L=a_{i j} \frac{\partial^{2}}{\partial x_{i} \partial x_{i}}+b_{i} \frac{\partial}{\partial x_{i}}+c,
$$

Received September 13, 1966. 
in which $a_{i j} \in C^{2}(\bar{\Omega})$ and $b_{i}, c \in C^{1}(\bar{\Omega})$. In this case Landis ${ }^{\circ}$ and Lavrentév proved our strong Cauchy uniqueness property. In particular, the latter showed that the exponent $\delta$ in (1.1) can be taken as an arbitrary number larger than 2 , if $\Gamma$ is a portion of a spherical surface. Their method could be said to give an explicit estimate expressing a relation between the solution and the Cauchy data. This rela. tion is called "well behaved" in the sense of F. John [6].

Now does the strong Cauchy uniqueness property hold for higher order elliptic equations? We can show this property if the dimension $N=2$ and if the elliptic operator has distinct characteristic roots. This is an extension of a result of Carleman [4]. In particular, for the second order elliptic operator in (1.2) the regularity assumptions on the coefficients can be relaxed in such a way

$$
a_{i j} \in C^{1}(\bar{\Omega}), b_{i} \text { and } c \in L^{\infty}(\bar{\Omega}),
$$

where the dimension $N=2$ and $L^{\infty}(\bar{\Omega})$ means a class of bounded measurable functions in $\Omega$. The method used in this note consists in establishing an energy integral estimates containing an adequate weight function.

From now on we consider in the 2 dimensional space with the coordinate $(x, y)$ and with the metric norm $r\left(=\left(x^{2}+y^{2}\right)^{1 / 2}\right)$. Let $\Gamma$ be a smooth curve containing the origin in its interior and let $\Omega$ be a domain whose boundary contains $\Gamma$. First we treat the solutions $u_{p}(p=1, \cdots, m)$ of the elliptic system

$$
\frac{\partial u_{p}}{\partial x}+\sum_{q=1}^{m} a_{p, q}(x, y) \frac{\partial u_{q}}{\partial y}+\sum_{q=1}^{m} b_{p, q}(x, y) u_{q}=0,
$$

where $a_{p, q} \in C^{1}(\bar{\Omega}), \quad b_{p, q} \in L^{\infty}(\bar{\Omega})$ and $u_{p} \in C^{1}(\bar{\Omega})$. We assume that all characteristic roots of $\lambda_{p}$ of the determinant

$$
\left|a_{p, q}-\lambda \delta_{p, q}\right|=0
$$

are distinct in $\bar{\Omega}$ and all imaginary parts of $\lambda_{p}$ are not zero in $\bar{\Omega}$. This system was considered by Carleman [4].

Theorem 1. There is a positive number $\delta$ such that if the solu- 
tions up of (1.3) satisfies

$$
u_{p}=o\left(\exp \left(-r^{-2 \delta}\right)\right) \quad(r \rightarrow 0) \quad p=1, \cdots, m
$$

along $\Gamma$, then $u \equiv 0$ in $\Omega$, where $\delta$ depends only on $\left\{a_{p, q}\right\}$ and $\Gamma$.

Next we consider the single elliptic equation of order $m$

$$
L \iota \equiv \sum_{i+j \leqq m} a_{i j} \frac{\partial^{i+j} u}{\partial x^{i} \partial y^{j}}=0 .
$$

In the same manner we assume that the characteristic roots of $L$ are distinct in $\bar{\Omega}$ and $a_{i j}(i+j=m)$ are in $C^{1}(\bar{\Omega})$, and the other coefficients are in $L^{\infty}(\bar{\Omega})$.

Theorem 2. Let $u$ be a solution of (1.5) in $\Omega$ and let ube in $C^{\prime \prime}(\bar{\Omega})$. Then there is a positive nimber $\delta$ depending only on $L$ and $\Gamma$. And if u satisfies

$$
\frac{\partial^{i+j} u}{\partial x^{i} \partial y^{j}}=o\left(\exp \left(-r^{-2 S}\right)\right) \quad(r \rightarrow 0) \quad i+j \leqq m-1
$$

along $\Gamma$, then $u \equiv 0$ in $\Omega$. In particular when $m=2$ and the coefficients of the principal part of $L$ are real, the nimber $\delta$ can be taken as an arbitrary number larger than 1 under the assumption that $\Gamma$ is a circular arc.

The proof of these theorems will be given in the last section. The author wishes to express his heartly thanks to Prof. S. Mizohata for his kind suggestions and encouragement.

2. First we prepare a mean value property for solutions of the elliptic equation. We consider in $r \leqq R_{0}(<1)$ the first order elliptic equation

$$
L u \equiv u_{x}+\lambda(x, y) u_{y}=f(x, y),
$$

where $u, \lambda \in C^{1}$ in $r \leqq R_{0}$ and the imaginary part of $\lambda$ is not zero in $r \leqq R_{0}$. We put $M(R)=\max _{r \leqq R}\left(1,|\lambda|,\left|\lambda_{x}\right|,\left|\lambda_{y}\right|\right)$ and $m(R)=\max _{r \leqq R}(1,|u|,|f|)$. And we denote the origin simply by 0 . Then we have

Lemma 1. When $\lambda(0)=i$ and $1<p<2$, the solution of (2.1) satisfies 


$$
\begin{aligned}
& |u(0)| \leqq C R^{(2 / p)-2} m(R)^{(2 / p)-1} M(R) \\
& \quad\left\{\left(\iint_{r \leqq R}|u|^{2} d x d y\right)^{1-(1 / p)}+\left(\iint_{r \leqq R}|f|^{2} d x d y\right)^{1-(1 / p)}\right\},
\end{aligned}
$$

where $C$ is a constant independent of $u, \lambda$ and $R$.

Proof. From now on we denote by $C_{1}, C_{2}, \cdots$ the constants independent of $u, \lambda$ and $R$.

We take the $C^{\infty}$ function $\phi(r)$ :

$$
\phi(r)=\left\{\begin{array}{lll}
1 & \text { in } & r \leqq R / 2 \\
0 & \text { in } & r>R
\end{array} \text { and }\left|\phi_{x}\right|,\left|\phi_{y}\right| \leqq C_{1} R^{-1}\right.
$$

Set $v=\phi u$. Then we see

$$
L v=L \phi \cdot u+\phi f
$$

and

$$
v_{x}+i v_{y}=L v+(\lambda(0)-\lambda) v_{y}
$$

Since $v$ has compact carrier, we have from (2.4)

$$
u(0)=-\frac{1}{2 \pi} \iint_{r \leqq R} \frac{L v+(\lambda(0)-\lambda) v_{y}}{x+i y} d x d y .
$$

And we see by (2.3)

$$
\begin{gathered}
\iint_{r \leqq R} \frac{L v}{x+i y} d x d y \mid \leqq C_{2}\left(R^{-1} M(R) \iint_{r \leqq R} \frac{|u|}{r} d x d y\right. \\
\left.+\iint_{r \leqq R} \frac{|f|}{r} d x d y\right) .
\end{gathered}
$$

By Green's formula we get

$$
\begin{array}{r}
\iint_{r \leqq R} \frac{\lambda(0)-\lambda}{x+i y} v_{y} d x d y=\iint_{r \leqq R} \frac{\lambda_{y} v}{x+i y} d x d y \\
+\iint_{r \leqq R} \frac{i(\lambda(0)-\lambda)}{(x+i y)^{2}} v d x d y .
\end{array}
$$

Hence we have

$$
\iiint_{r \leqq R} \frac{\lambda(0)-\lambda}{x+i y} v_{y} d x d y \mid \leqq C_{3} M(R) \iint_{r \leqq R} \frac{|u|}{r} d x d y .
$$

Combining (2.5), (2.6) and (2.7), we obtain 


$$
|u(0)| \leqq C_{4} R^{-1} M(R) \iint_{r \leqq R} \frac{1}{r}(|u|+|f|) d x d y .
$$

Applying Hölder's inequality we see

$$
\begin{aligned}
& \iint_{r \leqq R} \frac{1}{r} \frac{|u|}{m(R)} d x d y \\
& \quad \leqq\left(\iint_{r \leqq R} r^{-p} d x d y\right)^{1 / p}\left(\iint_{r \leqq R}\left(\frac{|u|}{m(R)}\right)^{q} d x d y\right)^{1 / q},
\end{aligned}
$$

where $\frac{1}{p}+\frac{1}{q}=1$. Since $1<p<2,2<q$ and $|u| \leqq m(R)$, it holds

$$
\iint_{r \leqq R} \frac{1}{r} \frac{|u|}{m(R)} d x d y \leqq \frac{1}{2-p} R^{2-p \mid p}\left(\iint_{r \leqq R}\left(\frac{|u|}{m(R)}\right)^{2} d x d y\right)^{1 / q} .
$$

That is,

$$
\begin{gathered}
\iint_{r \leqq R} \frac{|u|}{r} d x d y \leqq \frac{1}{2-p} R^{(2 / p)-1} m(R)^{(2 / p)-1} \\
\times\left(\iint_{r \leqq R}|u|^{2} d x d y\right)^{1-(1 / p)}
\end{gathered}
$$

Similarly we have

$$
\begin{gathered}
\iint_{r \leqq R} \frac{|f|}{r} d x d y \leqq \frac{1}{2-p} R^{(2 / p)-1} m(R)^{(2 / p)-1} \\
\times\left(\iint_{r \leqq R}|f|^{2} d x d y\right)^{1-(1 / p)} .
\end{gathered}
$$

Thus combining (2.8), (2.9) and (2.10), we have obtained the inequality (2.2).

Now we want to eliminate the assumption $\lambda(0)=i$ from Lemma 1 . We put $\lambda=\lambda_{1}+i \lambda_{2}$. And we transform the coordinate by

$$
\left(\begin{array}{l}
x^{\prime} \\
y^{\prime}
\end{array}\right)=\left(\begin{array}{cc}
1 & 0 \\
-\lambda_{1}(0) / \lambda_{2}(0) & 1 / \lambda_{2}(0
\end{array}\right)\left(\begin{array}{l}
x \\
y
\end{array}\right) \text {. }
$$

Then the operator $L$ in (2.1) is transformed into

$$
L^{\prime} u \equiv u_{x^{\prime}}+\left(-\lambda_{1}(0)+\lambda_{1}(x, y)+i \lambda_{2}(x, y)\right) u_{y^{\prime}} / \lambda_{2}(0) .
$$

If we denote the new metric norm by $\tilde{r}$, we see easily the following metric equivalent relation 


$$
B^{-1} r \leqq \tilde{r} \leqq B r,
$$

where $B$ is a positive constant depending only on $\lambda_{1}(0), \lambda_{2}(0)$.

Applying Lemma 1 to the operator $L^{\prime}$ in (2.11) we have

$$
\begin{aligned}
|u(0)| & \leqq C R^{(2 / p)-2} \widetilde{m}(R)^{(2 / p)-1} \widetilde{M}(R)\left\{\left(\iint_{\tilde{r} \leqq R}|u|^{2} d x^{\prime} d y^{\prime}\right)^{1-(1 / p)}\right. \\
& \left.+\left(\iint_{\tilde{r}_{\leqq}}|f|^{2} d x^{\prime} d y^{\prime}\right)^{1-(1 / p)}\right\},
\end{aligned}
$$

where $\widetilde{M}(R)=\max _{\tilde{r} \leqq R}\left(1,|\lambda|,\left|\lambda_{x^{\prime}}\right|,\left|\lambda_{y^{\prime}}\right|\right)$ and $\widetilde{m}(R)=\max _{\tilde{r} \leqq R}(1,|u|,|f|)$. We note that $\lambda_{y^{\prime}}=\lambda_{2}(0) \lambda_{y}$ and $\lambda_{x^{\prime}}=\lambda_{x}+\lambda_{1}(0) \lambda_{y}$. Then using (2.12), we get

$$
\widetilde{M}(R) \leqq M(B R) \max \left(1+\left|\lambda_{1}(0)\right|,\left|\lambda_{2}(0)\right|\right) .
$$

Similarly using the relation (2.12) for (2.13), we obtain

Proposition $\mathbb{1}$. For the solution of $(2.1)$ it holds that if $1<p<2$,

$$
\begin{gathered}
\mid u(0) \leqq k R^{(2 / p)-2} \widetilde{m}(B R)^{(2 / p)-1} M(B R)\left\{\left(\iint_{r \leqq B R}|u|^{2} d x d y\right)^{1-(1 / p)}\right. \\
\left.+\left(\iint_{r \leqq B r}|f|^{2} d x d y\right)^{1-(1 / p)}\right\}
\end{gathered}
$$

where $k=C \lambda_{2}(0)^{(1 / p)-1} \max \left(1+\left|\lambda_{1}(0)\right|,\left|\lambda_{2}(0)\right|\right)$ and $C$ is a constant independent of $u, \lambda, R$.

3. In this section we see how the behavior of the solution of (1.3) (or (1.5)) is influenced by the Cauchy data. Let us denote by $S_{d}$ an open disc with the center $(d / 2,0)$ and with the radius $d / 2$. We put $\Omega_{h}=\{0<x<h\} \cap S_{1}, \Gamma_{h}=\{0 \leqq x \leqq h\} \cap \partial S_{1}$ and $l_{h}=\{x=h\} \cap S_{1}$.

Lemma 2. If $u \in C^{1}\left(\bar{\Omega}_{a}\right)$ and $u=o\left(\exp \left(-r^{-2 \delta-\varepsilon}\right)\right) \quad(r \rightarrow 0)$ along $\Gamma_{a}$ for some positive numbers $\delta, \varepsilon$, then there is a function $v$ such that

$$
\begin{gathered}
v \in C^{0}\left(\bar{\Omega}_{a}\right) \text { and } v=u \text { on } \Gamma_{a} \\
v \in C^{1}\left(\bar{\Omega}_{a}-\{0\}\right) \text { and } \\
\int_{l_{k}}|v|^{2} d y, \int_{l_{h}}\left|v_{y}\right|^{2} d y \text { and } \int_{l_{k}}\left|v_{x}\right|^{2} d y \\
=o\left(\exp \left(-h^{-\delta}\right)\right) \quad(h \rightarrow 0)
\end{gathered}
$$


Proof. We take a $C^{\infty}$ function $\varphi(x)$ such that

$$
\int_{-\infty}^{\infty} \varphi(x) d x=1, \text { and the carrier of } \varphi \subset\{|x| \leqq 1\} \text {. }
$$

If we set

$$
f_{s}(x)=\int_{-2 s}^{2 s} \varphi((x-y) / s) \frac{1}{s} d y,
$$

we see that

$$
\begin{aligned}
& f_{s}(x) \in C^{\infty}(-\infty, \infty), \\
& f_{s}(x)=1 \text { for }|x| \leqq s \text { and } f_{s}(x)=0 \text { for }|x| \geqq 3 s, \\
& \left.\left|f_{s}(x)\right| \leqq 1 \text { and }\left|d f_{s}(x) / d x\right| \leqq c / s \text { ( } c \text { is independent of } s\right) .
\end{aligned}
$$

We put $k(x)=\exp \left(-x^{-\delta-(\varepsilon / 2)}\right)$ and write a curve $\Gamma^{\prime}$ such that

$$
\begin{array}{ll}
\Gamma^{\prime}: & x^{2}+(y+k(x))^{2}=x \text { for } y>0, \\
& x^{2}+(y-k(x))^{2}=x \text { for } y<0 .
\end{array}
$$

The curve $\Gamma^{\prime}$ is smooth and tangent to $S_{1}$ from the interior. We define a function $v(x, y)$ by

$$
v(x, y)=\left\{\begin{array}{l}
u\left(x, \sqrt{x-x^{2}}\right) f_{k(x) / 3}\left(y-\sqrt{x-x^{2}}\right) \text { for } y>0, \\
u\left(x,-\sqrt{x-x^{2}}\right) f_{k(x) / 3}\left(y+\sqrt{x+x^{2}}\right) \text { for } y<0 .
\end{array}\right.
$$

We see from (3.1), (3.2) that this function satisfies (i).

We have from (3.3)

$$
\left|v_{y}\right| \leqq\left\{\begin{array}{l}
c\left|u\left(x, \sqrt{x-x^{2}}\right)\right| / k(x), \\
c\left|u\left(x,-\sqrt{x-x^{2}}\right)\right| / k(x)
\end{array}\right.
$$

Let us note that

$$
u=o\left(\exp \left(-h^{-\delta-(\varepsilon / 2)}\right) \quad(h \rightarrow 0) \quad \text { on } \quad \Gamma_{a} .\right.
$$

Hence $u\left(x, \sqrt{x-x^{2}}\right) / k(x)=o(1) \quad(x \rightarrow 0)$. Thus we get

$$
\int_{l_{l k}}\left|v^{2}\right| d y, \int_{l_{h}}\left|v_{y}\right|^{2} d y=o\left(\exp \left(-h^{-\delta}\right)\right) \quad(h \rightarrow 0) .
$$

A computation shows for $y>0$

$$
v_{x}=\left\{u_{x}\left(x, \sqrt{x-x^{2}}\right)+\frac{1}{2} u_{y}\left(x, \sqrt{x-x^{2}}\right) \frac{1-2 x}{\sqrt{x-x^{2}}}\right\} \text {. }
$$




$$
\begin{aligned}
& f_{k(x) / 3}\left(y-\sqrt{x-x^{2}}\right) \\
& \quad+\frac{2 k^{\prime}(x)}{k(x)} u\left(x, \sqrt{x-x^{2}}\right)\left\{\varphi\left(\frac{3}{k(x)}\left(y-\sqrt{x-x^{2}}-\frac{2}{3} k(x)\right)\right)\right. \\
& \left.\quad+\varphi\left(\frac{3}{k(x)}\left(y-\sqrt{x-x^{2}}+\frac{2}{3} k(x)\right)\right)\right\} \\
& \quad+u\left(x, \sqrt{x-x^{2}}\right) \int_{-\frac{2}{3} k(x)}^{\frac{2}{3} k(x)}\left\{\varphi\left(\left(y-\sqrt{x-x^{2}}-z\right) \frac{3}{k(x)}\right) \frac{3}{k(x)}\right\}_{x} d z 。
\end{aligned}
$$

while,

$$
\begin{aligned}
& \left\{\varphi\left(\frac{3}{k(x)}\left(y-\sqrt{x-x^{2}}-z\right)\right) \frac{3}{k(x)}\right\}_{*} \\
& =-\frac{3 k^{\prime}(x)}{k(x)^{2}} \varphi\left(\frac{3}{k(x)}\left(y-\sqrt{x-x^{2}}-z\right)\right) \\
& -\frac{9}{k(x)^{3}}\left\{\frac{k(x)}{2} \frac{1-2 x}{\sqrt{x-x^{2}}}+k^{\prime}(x)\left(y-\sqrt{x-x^{2}}-z\right)\right\} \\
& \quad \times \varphi^{\prime}\left(\left(y-\sqrt{x-x^{2}}-z\right) \frac{3}{k(x)}\right) .
\end{aligned}
$$

Hence we have for $y>0$

$$
\begin{aligned}
\left|v_{x}\right| & \leqq \text { const. }\left\{\left|u_{x}\left(x, \sqrt{x-x^{2}}\right)\right|+\frac{1}{\sqrt{x}}\left|u_{y}\left(x, \sqrt{x-x^{2}}\right)\right|\right. \\
& +\mid u\left(x, \sqrt{\left.x-x^{2}\right)} \mid\left(\frac{\left|k^{\prime}(x)\right|}{k(x)}+\frac{1}{\sqrt{x}} \frac{1}{k(x)}+\frac{\left|k^{\prime}(x)\right|}{k(x)^{2}}\right) .\right.
\end{aligned}
$$

Similarly we have the same inequality for $y<0$. Thus we obtain

$$
\int_{t_{h}}\left|v_{x}\right|^{2} d y=o\left(\exp \left(-h^{-\delta}\right)\right) \quad(h \rightarrow 0) .
$$

Combining (3.4) and (3.5), we have completed the proof.

Now we consider in $\bar{\Omega}_{a}$ the $m$-th order elliptic equation (1.5). We can assume that the coefficient $a_{m, 0}$ equals identically 1 in $\overline{\Omega_{a}}$. Let us set

$$
v_{j}=\partial^{m-1} u / \partial x^{j-1} \partial y^{m-j} \quad j=1, \cdots, m,
$$

then we see

$$
\frac{\partial}{\partial x}\left(\begin{array}{c}
v_{1} \\
\vdots \\
v_{m}
\end{array}\right)+\left(\begin{array}{ccccc}
0 & -1 & \ddots & \ddots & 0 \\
\vdots & 0 & \ddots & -1 \\
a_{0, m} & \cdots & a_{m-2,2} & a_{m-1,1}
\end{array}\right) \frac{\partial}{\partial y}\left(\begin{array}{c}
v_{1} \\
\vdots \\
v_{m}
\end{array}\right)=\left(\begin{array}{c}
0 \\
\vdots \\
0 \\
\mathcal{B} u
\end{array}\right)
$$


where $\mathscr{B}$ is a differential operator of order $m-2$ whose coefficients are in $L^{\infty}\left(\overline{\Omega_{a}}\right)$. We write (3.6) in the following form:

Set

$$
v_{x}+H v_{y}=g \text {. }
$$

$$
N=\left(\begin{array}{l}
1 \cdots \cdots 1 \\
\lambda_{1} \cdots \cdots \lambda_{m} \\
\lambda_{1}^{m-1} \cdots \lambda_{m}^{m-1}
\end{array}\right), \quad D=\left(\begin{array}{rrrr}
-\lambda_{1} & & & 0 \\
& \ddots & & \\
0 & \ddots & -\lambda_{m}
\end{array}\right),
$$

where $\lambda_{j}$ are characteristic roots of (1.5), and $w=N^{-1} v$, then we see easily

$$
w_{x}+D w_{y}=N^{-1}\left(g-N_{x} w-H N_{y} w\right) .
$$

From now on we denote $\int_{l_{h}}|u(h, y)|^{2} d y$ by $\|u(h)\|^{2}$ and $\int_{l_{h}} u(h, y) \overline{v(h, y)} d y$ by $[u(h), v(h)]$.

Proposition 2. Let $u$ be a solution of the elliptic equation (1.5) in $\Omega_{a}$ and $u$ be in $C^{m}\left(\bar{\Omega}_{a}\right)$. If for some $\varepsilon>0, \delta>1$

$$
\frac{\partial^{i+j} u}{\partial x^{i} \partial y^{j}}=o\left(\exp \left(-r^{-2 \delta^{-\varepsilon}}\right)\right) \quad(r \rightarrow 0, i+j \leqq m-1) \quad \text { on } \quad \Gamma_{a},
$$

then we have ${ }^{1)}$

$$
\int_{0}^{h}\left\|\frac{\partial^{i+j} u}{\partial x^{i} \partial y^{j}}\right\|^{2} d x=o\left(\exp \left(-h^{-\delta}\right)\right) \quad(h \rightarrow 0, i+j \leqq m-1) .
$$

Proof. We reduce the equation (1.5) into the form (3.7). And we put

$$
M_{i} \equiv \frac{\partial}{\partial x}-\lambda_{i}(x, y) \frac{\partial}{\partial y}, \quad i=1, \cdots, m,
$$

where $\lambda_{i}=\lambda_{i 1}+i \lambda_{i 2}$ and $\lambda_{i 2} \neq 0$ in $\bar{\Omega}_{a}$.

If $\psi(x)$ is a function of one variable and $\psi(x) \in C^{1}[a, b]$, then it holds

$$
\int_{a}^{b}|\psi(x)|^{2} d x \leqq 2(b-a)\left((b-a) \int_{a}^{b}\left|\psi^{\prime}(x)\right|^{2} d x+|\psi(a)|^{2}\right),
$$

using successively this inequality, we have for the operator $\mathscr{B}$ in (3.6)

1) When all characteristic roots of (1.5) are real in $\bar{\Omega}_{a}$ (hyperbolic), this proposition holds also. 
$\|(\mathscr{B} u)(h)\| \leqq$ const. $\left\{\sum_{j=1}^{m}\left\|w_{j}(h)\right\|+\sum_{i+j \leqq m-2} \mid \frac{\partial^{i+j} u}{\partial x^{i} \partial y^{j}}\left(P_{h}\right)\right\}$, where $P_{h} \in \Gamma_{a}$ and $P_{h}=\left(h, \sqrt{h-h^{2}}\right)$. Hence we see

$$
\begin{gathered}
\left\|M_{i} w_{i}(h)\right\|^{2} \leqq \text { const. }\left(\sum_{j=1}^{m}\left\|w_{j}(h)\right\|^{2}+\exp \left(-h^{-\delta-(\varepsilon / 2)}\right)\right) . \\
i=1, \cdots, m .
\end{gathered}
$$

From Lemma 2 there are functions $\widetilde{\boldsymbol{w}}_{i} \in C^{0}\left(\overline{\Omega_{a}}\right)(i=1, \cdots, m)$ such that

(3.10) $\widetilde{w}_{i}=w_{i}$ on $\Gamma_{a}$ and $\widetilde{w}_{i} \in C^{1}\left(\overline{\Omega_{a}}-\{0\}\right)$,

(3.11) $\left\|\widetilde{w}_{i}(h)\right\|^{2},\left\|\widetilde{w}_{i y}(h)\right\|^{2}$ and $\left\|\widetilde{w}_{i x}(h)\right\|^{2}$

$$
=o\left(\exp \left(-h^{-\delta-(\varepsilon / 3)}\right)\right)(h \rightarrow 0) \text {. }
$$

Now we put $z_{i}=w_{i}-\widetilde{w}_{i}$ and $\varphi_{n}(x)=\left(x+\frac{1}{n}\right)^{-n}$. And let us denote by $c_{i}(i=1,2, \cdots)$ the positive constants independent of $n$ and $h$. We have by [3] and [10]

$$
\begin{gathered}
\int_{\delta}^{h} \varphi_{n}(x)^{2}\left\|\frac{\partial z_{i}}{\partial x}-\lambda_{i} \frac{\partial z_{i}}{\partial y}\right\|^{2} d x \\
\geqq \frac{c_{1}}{n}\left(1-c_{1}\left(h+\frac{1}{n}\right)\right) \int_{\delta}^{h} \varphi_{n}^{\prime}(x)^{2}\left\|z_{i}\right\|^{2} d x-I_{n}(\delta, h) \\
\left(i f\left(h+\frac{1}{n}\right)<c_{1}^{-1}\right),
\end{gathered}
$$

where $\quad\left|I_{n}(\delta, h)\right| \leqq n \varphi_{n}(\delta)^{2}\left\|z_{i}(\delta)\right\|^{2}+\varphi_{n}(\delta)^{2}\left|\left[z_{i}(\delta), \lambda_{i 2} z_{i y}(\delta)\right]\right|$

$$
+\varphi_{n}(h)^{2}\left|\left[z_{i}(h), \lambda_{i 2} z_{i y}(h)\right]\right| \text {. }
$$

We see from (3.11) that $\left\|z_{i}(\delta)\right\| \rightarrow 0,\left\|z_{i y}(\delta)\right\| \rightarrow 0$ as $\delta \rightarrow 0$. Thus,

$$
\begin{aligned}
& \int_{0}^{h} \varphi_{n}(x)^{2}\left\|\frac{\partial z_{i}}{\partial x}-\lambda_{i} \frac{\partial z_{i}}{\partial y}\right\|^{2} d x \\
& \geqq \frac{c_{1}}{n}\left(1-c_{1}\left(h+\frac{1}{n}\right)\right) \int_{0}^{h} \varphi^{\prime}(x)^{2}\left\|z_{i}\right\|^{2} d x \\
& -\varphi_{n}(h)^{2}\left|\left[z_{i}(h), \lambda_{i 2} z_{i y}(h)\right]\right| .
\end{aligned}
$$

Since $\varphi_{n}(h)^{2}\left|\left[z_{i}(h), \lambda_{i 2} z_{i y}(h)\right]\right| \leqq c_{2}\left(h+\frac{1}{n}\right)^{-2 n}$, taking $h+\frac{1}{n}<\frac{1}{2 c_{1}}$ in 
(3.12), we get

$$
c_{2}\left(h+\frac{1}{n}\right)^{-2 n}+\int_{0}^{h} \varphi_{n}(x)^{2}\left\|M_{i} z_{i}\right\|^{2} d x \geqq \frac{c_{1}}{2 n} \int_{0}^{h} \varphi^{\prime}{ }_{n}(x)^{2}\left\|z_{i}\right\|^{2} d x .
$$

We substitute $z_{i}=w_{i}-\widetilde{\mathfrak{w}}_{i}$ into the inequality. Then we see

$$
\begin{aligned}
c_{2}(h & \left.+\frac{1}{n}\right)^{-2 n}+2 \int_{0}^{h} \varphi_{n}(x)^{2}\left\|M_{i} w_{i}\right\|^{2} d x+2 \int_{0}^{h} \varphi_{n}(x)^{2}\left\|M_{i} \widetilde{\widetilde{v}}_{i}\right\|^{2} d x \\
& +\frac{c_{1}}{2 n} \int_{0}^{h} \varphi^{\prime}{ }_{n}(x)^{2}\left\|\widetilde{w}_{i}\right\|^{2} d x \geqq \frac{c_{1}}{4 n} \int_{0}^{h} \varphi^{\prime}{ }_{n}(x)^{2}\left\|w_{i}\right\|^{2} d x .
\end{aligned}
$$

From (3.9) and (3.11) we have

$$
\begin{aligned}
c_{3} n^{2 n+2} & \left(\frac{h}{2}+\frac{1}{n}\right)^{2 n+2} \exp \left(-h^{-\delta-(\varepsilon / 3)}\right) \\
& +c_{4} n^{-1}\left(h+\frac{1}{n}\right)^{-2 n}\left(\frac{h}{2}+\frac{1}{n}\right)^{2 n+2} \geqq \sum_{i=1}^{m} \int_{0}^{h / 2}\left\|\boldsymbol{r}_{i}\right\|^{2} d x .
\end{aligned}
$$

Let us take $h+\frac{1}{n}$ sufficiently small and $n h$ sufficiently large. As an easy computation shows, in order to prove

$$
\int_{0}^{h / 2}\left\|w_{i}\right\|^{2} d x=o\left(\exp \left(-\left(\frac{h}{2}\right)^{-\delta}\right)\right) \quad(h \rightarrow 0, i=1, \cdots, m),
$$

it is sufficient to show that we can choose $n$ in such a way that

$$
n^{2 n+2} \leqq \exp \left(\left(\frac{h}{2}\right)^{-\delta-\varepsilon_{1}}\right)
$$

$$
\left(\frac{h n+2}{2 h n+2}\right)^{2 n} \leqq \exp \left(-\left(\frac{h}{2}\right)^{-\delta-\varepsilon^{\prime}}\right) \quad \varepsilon_{1}, \varepsilon^{\prime}>0
$$

where $\varepsilon_{1}$ is a given number and $\varepsilon^{\prime}$ will be determined later. The inequalities (3.14) are verified if the following inequalities are satisfied

$$
n^{1+\bar{\varepsilon}} \leqq\left(\frac{h}{2}\right)^{-\delta-\varepsilon_{1}} \quad \bar{\varepsilon}>0
$$

$$
2 n \log \frac{2}{3} \leqq-\left(\frac{h}{2}\right)^{-\delta-\varepsilon^{\prime}}
$$

Here we can take $\bar{\varepsilon}$ arbitrarily small if $n$ is sufficiently large. If we 
take $\bar{\varepsilon}$ sufficiently small, there is a positive number $\varepsilon^{\prime}$ such that

$$
\frac{1}{2 \log \frac{3}{2}}\left(\frac{2}{h}\right)^{\delta+\varepsilon^{\prime}}<\left(\frac{2}{h}\right)^{\left(\delta+\varepsilon_{1}\right) /(1+\varepsilon)} .
$$

Let us take $n$ in such a way that

$$
\frac{1}{2 \log \frac{3}{2}}\left(\frac{2}{h}\right)^{\delta+\varepsilon^{\prime}}<n<\left(\frac{2}{h}\right)^{\left(\delta+\varepsilon_{1}\right) /(1+\varepsilon)} .
$$

Then (3.15) is satisfied and $n h \rightarrow \infty$ as $h \rightarrow 0$. Thus we have proved (3.13).

Now we take an open disk $S_{1 / 2}$ in $S_{1}$. For the point $(x, y)$ in $S_{1 / 2}$ we denote by $r_{1}(x, y)$ the radius of a circle tangent to $S_{1}$ whose center is $(x, y)$. It is easily seen that there is a constant $c$ such that

$$
c^{-1} x \leqq r_{1}(x, y) \leqq c x .
$$

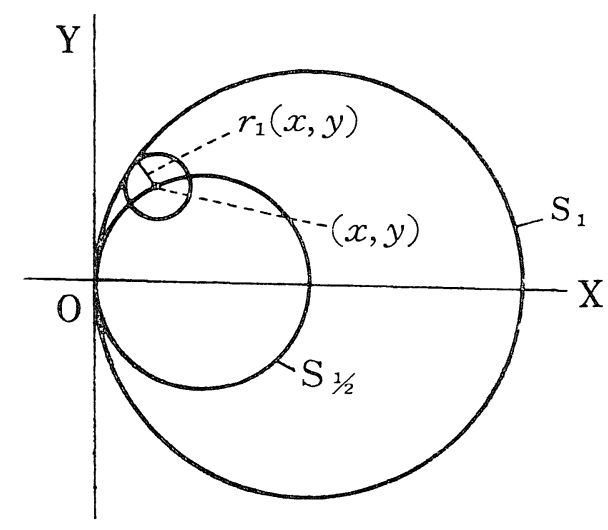

Proposition 3. Let $u$ be a solution of the elliptic equation (1.5) in $\Omega_{a}$ and $u$ be in $C^{m}\left(\bar{\Omega}_{a}\right)$. Then if for some $\varepsilon>0, \delta>1$

$$
\frac{\partial^{i+j} u}{\partial x^{i} \partial y^{j}}=o\left(\exp \left(-r^{-2 \delta-\varepsilon}\right)\right) \quad(r \rightarrow 0, i+j \leqq m-1) \quad \text { on } \quad \Gamma_{a},
$$

we have in $S_{1 / 2} \cap \Omega_{a}$

$$
\frac{\partial^{i+j} u}{\partial x^{i} \partial y^{j}}=o\left(\exp \left(-r^{-\delta}\right)\right) \quad(r \rightarrow 0, i+j \leqq m-1) .
$$


Proof. We reduce the equation (1.5) into the form (3.7). We set $-2 \delta-\varepsilon=-2\left(\delta+\frac{\varepsilon}{3}\right)-\frac{\varepsilon}{3}$. And we regard $\delta+\frac{\varepsilon}{3}$ as new $\delta$. Then by Proposition 2 we have

$$
\begin{aligned}
& \int_{0}^{h}\left\|\frac{\partial^{i+j} u}{\partial x^{i} \partial y^{j}}\right\|^{2} d x=o\left(\exp \left(-h^{-\delta-(\varepsilon / 3)}\right)\right) \\
&(h \rightarrow 0, i+j \leqq m-1) .
\end{aligned}
$$

This means that

$$
\int_{0}^{h}\left\|w_{i}\right\|^{2} d x=o\left(\exp \left(-h^{-\delta-(\varepsilon / 3)}\right)\right) \quad(h \rightarrow 0)
$$

and for $\mathcal{B} u$ in $(3.6)$

$$
\int_{0}^{h}\|\mathscr{B} u\|^{2} d x=o\left(\exp \left(-h^{-\delta-(\varepsilon / 3)}\right)\right) \quad(h \rightarrow 0) .
$$

We apply Proposition $I$ for the equation (3.7) in a disk with center $(x, y)$ and with radius $r_{1}(x, y)$. In (2.14) we can take $B$ and $k$ as fixed constant in $S_{1 / 2}$. And from the behavior of $u$ at the origin it is assumed that $\widetilde{m}(B R) \leqq 1$. Putting $p=3 / 2$ in (2.14), we have from (2.14) and (3.7)

$$
\begin{aligned}
& \left|w_{i}\left(x^{(0)}, y^{(0)}\right)\right| \\
& \leq C R^{-2 / 3}\left\{\left(\iint_{\sqrt{(x-x(0))^{2}+(y-y(0))^{2}} \leq B R}\left|w_{i}\right|^{2} d x d y\right)^{1 / 3}\right. \\
& \quad+\left(\iint_{\sqrt{(x-x(0))^{2}+(y-y(0))^{2}} \leq B R} \sum_{j=1}^{m}\left|w_{i}\right|^{2} d x d y\right. \\
& \left.\left.\quad+\iint_{\sqrt{(x-x(0))^{2}+(y-y(0))^{2}} \leq B R}|\mathscr{B u}|^{2} d x d y\right)^{1 / 3}\right\},
\end{aligned}
$$

where $\left(x^{(0)}, y^{(0)}\right) \in S_{1 / 2}, B R=r_{1}\left(x^{(0)}, y^{(0)}\right)$ and $C$ is a constant independent of $(x, y)$. Thus we get

$$
\begin{aligned}
\mid w_{i}(x, y) i \leqq & C r(x, y)^{-2 / 3}\left\{\left(\int_{0}^{x+r_{1}(x, y)}\left\|w_{i}\right\|^{2} d t\right)^{1 / 3}\right. \\
& \left.+\left(\int_{0}^{x+r_{1}(x, y)} \sum_{j=1}^{m}\left\|w_{j}\right\|^{2} d t+\int_{0}^{x+r_{1}(x, y)}\|\mathscr{B} u\|^{2} d t\right)^{1 / 3}\right\},
\end{aligned}
$$

combining (3.18) and (3.19), we obtain

$$
\left|w_{i}(x, y)\right| \leqq \text { const. }\left(c^{-1} x\right)^{-2 / 3} \exp \left(-(c x+x)^{-\delta-(\varepsilon / 3)}\right)
$$


Hence we have

$$
\leqq \text { const. } \exp \left(-x^{-\delta-(\varepsilon / 4)}\right) \text {. }
$$

$$
\left|\frac{\partial^{m-1} u}{\partial x^{i} \partial y^{j}}\right| \leqq \text { const. } \exp \left(-r^{-\delta-(\varepsilon / 4)}\right) \quad i+j \leqq m-1 .
$$

Let us denote by $l_{(x, y)}$ the line connecting the origin and the point $(x, y)$. Then using the mean value theorem on $l_{(x, y)}$ we see

$$
\left|\frac{\partial^{m-2} u}{\partial x^{i} \partial y^{j}}(x, y)\right| \leqq r \sum_{i+j \leqq m-1}\left|\frac{\partial^{m-1} u}{\partial x^{i} \partial y^{j}}\left(x^{(i)}, y^{(j)}\right)\right|,
$$

where $\left(x^{(i)}, y^{(j)}\right) \in l_{(x, y)}$. Hence from (3.20) we get for $\partial^{m-2} u / \partial x^{i} \partial y^{j}$ the same inequality as in (3.20). Repeating this process we have obtaind (3.17).

We put for $d>0 \quad \Omega_{h, d}=\{0<x<h\} \cap S_{d}$ and $\Gamma_{h, d}=\{0 \leqq x \leqq h\} \cup \partial S_{d}$. Then the following corollary holds. This proof is reduced to Proposition 2 by an adequate coordinate transformation.

Corollary 1. Let $u$ be a solution of the elliptic equation (1.5) in $\Omega_{a, d}$ and $u$ be in $C^{n}\left(\bar{\Omega}_{a, d}\right)$. Then if for some $\varepsilon>0, \delta>1$

$$
\frac{\partial^{i+j} u}{\partial x^{i} \partial y^{j}}=o\left(\exp \left(-r^{-2 \delta-\varepsilon}\right)\right) \quad(r \rightarrow 0, i+j \leqq m-1) \quad \text { on } \quad \Gamma_{a, d},
$$

we have in $\Omega_{a, d / 2}$

$$
\frac{\partial^{i+j} u}{\partial x^{i} \partial y^{j}}=o\left(\exp \left(-r^{-\delta}\right)\right) \quad(r \rightarrow 0, \quad i+j \leqq m-1) .
$$

In the case of elliptic system (1.3) the reduced equation (3.7) has $g \equiv 0$. Thus we have in particular.

Corollary 2. Let $\left\{u_{p}\right\}$ be solutions of the elliptic system (1.3) in $\Omega_{a, d}$ and $\left\{u_{p}\right\}$ be in $C^{1}\left(\bar{\Omega}_{a, d}\right)$. Then if for some $\varepsilon>0, \delta>1$

$$
u_{p}=o\left(\exp \left(-r^{-2 \delta-\varepsilon}\right)\right) \quad(r \rightarrow 0,1 \leqq p \leqq m) \quad \text { on } \quad \Gamma_{a, d},
$$

we have in $\Omega_{a, d / 2}$

$$
u_{p}=o\left(\exp \left(-r^{-\delta}\right)\right) \quad(r \rightarrow 0,1 \leqq p \leqq m) .
$$

4. We consider the equation (1.3) and (1.5) in $\overline{\Omega_{a, d}}$. 
Proposition 4. There is a constant $\delta$ such that if $u$ is in $C^{m}\left(\bar{\Omega}_{a, d}\right)$ and is a solution of (1.5) in $\Omega_{a, d}$ satisfying

$$
\frac{\partial^{i+j} u}{\partial x^{i} y^{j}}=o\left(\exp \left(-r^{-\delta}\right)\right) \quad(r \rightarrow 0, i+j \leqq m-1) \quad \text { in } \quad \Omega_{a, d}
$$

then $u$ vanishes identically in $\Omega_{a, d}$. Here $\delta$ is independent of $u$.

Before proving this proposition we prepare some lemmas. We consider the next transformation from $(x, y)$-plane to $(\theta, \rho)$-plane

$$
\rho=r^{2} / x, \theta=\tan ^{-1}(y / x) .
$$

Let us denote by $R_{d}$ an open rectangular in $(\theta, \rho)$-plane such as

$$
-\pi / 2<\theta<\pi / 2,0<\rho<d .
$$

Then the transformation (4.2) maps $S_{d}$ onto $R_{d}$. Further this transformation and its inverse are $C^{\infty}$. We see

$$
\left(\begin{array}{ll}
\theta_{x} & \theta_{y} \\
& \\
\rho_{x} & \rho_{y}
\end{array}\right)=\left(\begin{array}{cc}
-\frac{1}{\rho} \tan \theta & 1 / \rho \\
1-\tan ^{2} \theta & 2 \tan \theta
\end{array}\right) .
$$

We eliminate the part $\rho=d$ from the boundary of $R_{d}$ and denote the remainder by $\partial R_{d}$. And let us put $\bar{R}_{d}=R_{d}+\partial R_{d}$. For the function $f(x, y)$ in $\overline{S_{d}}$ we define a function $\tilde{f}(\theta, \rho)$ in $\bar{R}_{d}$ by

$$
\tilde{f}(\theta, \rho)=\left\{\begin{array}{lll}
f(\theta, \rho) & \text { for } & (\theta, \rho) \in \partial R_{d}, \\
f(0,0) & \text { for } & (\theta, \rho) \in \partial R_{d} .
\end{array}\right.
$$

Lemma 3. If $f(x, y) \in C^{1}\left(\bar{S}_{d}\right)$, then $\tilde{f}(\theta, \rho) \in C^{1}\left(\bar{R}_{d}\right)$.

Since it is easily verified, we omit the proof. From now on we denote $\widetilde{f}$ by $f$ for simplicity. We consider the next equation in $S_{d}$

$$
u_{x}+\lambda u_{y} \equiv F,
$$

where $u \in C^{1}\left(\overline{S_{d}}\right), \lambda \in C^{1}\left(\overline{S_{d}}\right)$ and the imaginary part of $\lambda \neq 0$ in $\overline{S_{d}}$. Now by (4.3) the equation is transformed into

$$
\left(\rho_{x}+\lambda \rho_{y}\right) u_{\rho}+\left(\theta_{x}+\lambda \theta_{y}\right) u_{\theta} \equiv F .
$$

Lemma 4. If we set $f(\theta, \rho)=\left|\rho_{x}+\lambda \rho_{y}\right|^{2} \cos ^{4} \theta$, then we see $f \in C^{1}\left(\bar{R}_{d}\right)$ and there is a positive constant $m$ such that $f>m$ in $\bar{R}_{d}$. 
Proof. Since $f=\left|\cos ^{2} \theta+2 \lambda \sin \theta \cos \theta-\sin ^{2} \theta\right|^{2}$, there is a required $m$. And we see by Lemma $3 f \in C^{1}\left(\bar{R}_{d}\right)$.

Now the equation (4.5) is reduced to

$$
u_{\rho}+f^{-1} \cos ^{4} \theta\left(\rho_{x}+\bar{\lambda} \rho_{y}\right)\left(\theta_{x}+\lambda \theta_{y}\right) u_{\theta} \equiv f^{-1} \cos ^{4} \theta\left(\rho_{x}+\bar{\lambda} \rho_{y}\right) F .
$$

Setting this equation into the form

$$
u_{\rho}+\frac{1}{\rho}(Q+i P) u_{\theta} \equiv G,
$$

we have from (4.3)

$$
\begin{aligned}
P(\theta, \rho)= & \lambda_{2} f^{-1} \cos ^{2} \theta \\
Q(\theta, \rho)= & f^{-1} \cos \theta\left\{\sin \theta\left(\sin ^{2} \theta-\cos ^{2} \theta\right)\right. \\
& \left.+\lambda_{1}\left(\cos ^{3} \theta-3 \cos \theta \sin ^{2} \theta\right)+2|\lambda|^{2} \cos ^{2} \theta \sin \theta\right\},
\end{aligned}
$$

where $\lambda=\lambda_{1}+i \lambda_{2}$. By Lemma 3 and Lemma 4 , we see $P, Q \in C^{1}\left(\bar{R}_{d}\right)$. Let us set

$$
\alpha(\theta, \rho)=Q_{\theta}-\frac{Q P_{\theta}}{P}-1
$$

Then we have

Lemma 5. $Q P_{\theta} / P$ is continuous in $\bar{R}_{d}$. And in particular when $\lambda(0)= \pm i, a(\theta, \rho) \rightarrow 0$ uniformly in $\theta$ as $\rho \rightarrow 0$.

Proof. As we easily see from (4.8), $Q P_{\theta}=f^{-1} \cos ^{2} \theta \times$ (continuous function). Then, in view of $\left|\lambda_{2}\right| \geq c(>0)$, the first assertion follows. If we note $\lambda(\theta, 0)=i, \lambda_{\theta}(\theta, 0)=0$ and that $f(\theta, 0)=1, f_{\theta}(\theta, 0)=0$, we have $\alpha(\theta, 0)=0$. Since $\alpha(\theta, \rho)$ is continuous in $\bar{R}_{d}$, we have completed the proof.

From now on we denote by \|\| a $L^{2}$ norm with respect to $\theta(|\theta| \leqq \pi / 2)$. And we define a weight function by

$$
\phi_{n}(\rho)=\exp \left(n \rho^{-\delta}\right) \text {. }
$$

Proposition 5. Suppose that $\lambda \in C^{1}\left(\overline{S_{d}}\right)$ and the imaginary part of $\lambda \neq 0$ in $\overline{S_{d}}$. Then there is a positive number $\delta$ depending only on $\lambda$. And if $u \in C^{1}\left(\overline{S_{d}}\right)$ and satisfies for some positive $\varepsilon$

$$
u=o\left(\exp \left(-r^{-\delta-\varepsilon}\right)\right) \quad(r \rightarrow 0) \quad \text { in } S_{d}
$$


then we have for sufficiently small $h$

$$
\begin{aligned}
& \int_{0}^{h} \phi_{n}^{2}\left\|u_{\rho}+\frac{1}{\rho}(Q+i P) u_{\vartheta}\right\|^{2} d \rho \\
& \quad \geqq c_{2} n \int_{0}^{h} \frac{\phi_{n}^{2}}{\rho^{\delta+2}}\|u\|_{1}^{2} d \rho-\frac{c_{1}}{h} \exp \left(2 n h^{-\delta}\right),
\end{aligned}
$$

where $c_{1}, c_{2}, \cdots$ are positive constants independent of $n$.

Proof. We set

$$
\begin{aligned}
\phi \iota_{\rho} & +\frac{1}{\rho} \phi(Q+i P) u_{\theta} \\
= & {\left[(\phi u)^{\prime}+\frac{1}{\rho} \phi Q u_{\theta}\right]+\left[\frac{1}{\rho} \phi i P \iota_{\theta}-\phi^{\prime} \iota\right] . }
\end{aligned}
$$

Here we omit $\phi_{n}(\rho)$ by $\phi$ and $d / d \rho$ by '(prime). In order to estimate the $L^{2}$ norm of the left side of (4.11) it is necessary to estimate following four terms.

$$
\begin{aligned}
&-\int_{0}^{h}( {\left.\left[(\phi u)^{\prime}, \phi^{\prime} u\right]+\left[\phi^{\prime} u,(\phi u)^{\prime}\right]\right) d \rho } \\
& \geqq \int_{0}^{h}\left(\phi \phi^{\prime \prime}-\phi^{\prime 2}\right)\|u\|^{2} d \rho \\
&= \int_{0}^{h} \frac{n \delta(\delta+1)}{\rho^{\delta+2}} \phi^{2}\|u\|^{2} d \rho . \\
&-\int_{0}^{h}\left(\left[\frac{1}{\rho} \phi Q u u_{\theta}, \phi^{\prime} u\right]+\left[\phi^{\prime} u, \frac{1}{\rho} \phi Q u u_{\theta}\right]\right) d \rho \\
&=\int_{0}^{h} \frac{\phi \phi^{\prime}}{\rho}\left[Q_{\theta} u, u\right] d \rho . \\
& \int_{0}^{h}\left(\left[(\phi u)^{\prime}, \frac{1}{\rho} \phi i P_{u}\right]+\left[\frac{1}{\rho} \phi i P u \iota_{\theta},(\phi u)^{\prime}\right]\right) d \rho \\
&= {\left[\phi u, \frac{1}{\rho} \phi i P u u_{\theta}\right](h)+\int_{0}^{h}\left[\phi u, \frac{i}{\rho^{2}} \phi P u_{\theta}\right] d \rho } \\
& \quad-\int_{0}^{h}\left[\phi u, \frac{1}{\rho} \phi i P^{\prime} u_{\theta}\right] d \rho \\
&+\int_{0}^{h}\left[-\frac{1}{\rho} P_{\theta} \phi u,(\phi u)^{\prime}+\frac{1}{\rho} \phi Q u u_{\theta}\right] d \rho \\
&+\int_{0}^{h}\left[\frac{1}{\rho} \phi i P_{\theta} u, \frac{1}{\rho} \phi Q u_{\theta}\right] d \rho .
\end{aligned}
$$




$$
\int_{0}^{h}\left(\left[\frac{1}{\rho} \phi Q u_{\theta}, \frac{1}{\rho} \phi i P u_{\theta}\right]+\left[\frac{1}{\rho} \phi i P u_{\theta}, \frac{1}{\rho} \phi Q u_{\theta}\right]\right) d \rho=0 .
$$

We put the right side of (4.15) into $\sum_{j=1}^{5} I_{j}$. Then we see

$$
\begin{aligned}
I_{2}= & \int_{0}^{h}\left[\frac{\phi u}{\rho}, \frac{1}{\rho} \phi i P u_{\theta}-\phi^{\prime} u\right] d \rho+\int_{0}^{h} \frac{\phi \phi^{\prime}}{\rho}[u, u] d \rho, \\
I_{3}= & -\int_{0}^{h}\left[\phi u, \frac{P^{\prime}}{P}\left(\frac{1}{\rho} \phi i P u_{\theta}-\phi^{\prime} u\right)\right] d \rho+\int_{0}^{h} \phi \phi^{\prime}\left[\frac{P^{\prime}}{P} u, u\right] d \rho, \\
I_{5}= & -\int_{0}^{h}\left[\frac{1}{\rho} \phi u, \frac{P_{\theta} Q}{P}\left(\frac{1}{\rho} \phi i P u_{\theta}-\phi^{\prime} u\right)\right] d \rho \\
& -\int_{0}^{h} \frac{\phi \phi^{\prime}}{\rho}\left[\frac{P_{\theta} Q}{P} u, u\right] d \rho .
\end{aligned}
$$

Here we note $P_{\theta} Q / P$ is bounded by Lemma 5. Applying Cauchy's inequality to each term we get

$$
\begin{aligned}
(4.14) & +(4.15) \geqq \int \frac{\phi \phi^{\prime}}{\rho}\left[\left(Q_{\theta}+1-\frac{P_{\theta} Q}{P}\right) u, u\right] d, 0 \\
& -\frac{c_{1}}{h} \exp \left(2 n h^{-\delta}\right)-c_{2} \int_{0}^{h} \frac{\phi^{2}}{\rho^{2}}\|u\|^{2} d \rho \\
& -c_{3} \int_{0}^{h} \phi^{2}\left\|u u^{2}\right\| d \rho-c_{4} \int_{0}^{h}\left|\phi \phi^{\prime}\right|\|u\|^{2} d \rho \\
& -\int_{0}^{h}\left\{\left\|(\phi u)^{\prime}+\frac{1}{\rho} \phi Q u u_{\theta}\right\|^{2}+\left\|\frac{1}{\rho} \phi P u_{\theta}-\phi^{\prime} u\right\|^{2}\right\} d \rho .
\end{aligned}
$$

We put $M=\max || Q_{\theta}+1-\frac{P_{\theta} Q}{P} \mid$. Then combining (4.13) and (4.17) we obtain

$$
\begin{aligned}
& \int_{0}^{h} \phi^{2}\left\|u_{\rho}+\frac{1}{\rho}(Q+i P) u_{\theta}\right\|^{2} d \rho \\
& \geqq n \delta(\delta+1-M) \int_{0}^{h} \frac{\phi^{2}}{\rho^{\delta+2}}\|u\|^{2} d \rho-\frac{c_{1}}{h} \exp \left(2 n h^{-\delta}\right) \\
& \quad-c_{2} \int_{0}^{h} \frac{\phi^{2}}{\rho^{2}}\|u\|^{2} d \rho-c_{3} n \delta \int_{0}^{h} \frac{\phi^{2}}{\rho^{\delta+1}}\|u\|^{2} d \rho
\end{aligned}
$$

Thus taking $\delta>M-1$ and $h$ sufficiently small, we have completed the proof.

Corollary 3. If we assume $\lambda(0)= \pm i$ in Proposition 5 , then we 
can take $\delta$ as an arbitrary number larger than 1.

Proof. We see from Lemma $5 Q_{\theta}+1-\frac{Q P_{\theta}}{P}=2+\alpha(\theta, \rho)(\alpha(\theta, \rho) \rightarrow 0$ uniformly in $\theta$ as $\rho \rightarrow 0$ ). Thus combining (4.13) and (4.17) we have

$$
\begin{aligned}
& \int_{0}^{h} \phi^{2}\left\|u_{\rho}+\frac{1}{\rho}(Q+i P) u u_{\theta}\right\|^{2} d \rho \\
& \quad \geqq \int_{0}^{h} \frac{n \delta(\delta+1)}{\rho^{\delta+2}} \phi^{2}\|u\|^{2} d \rho-\int_{0}^{h} \frac{\left|\phi \phi^{\prime}\right|}{\rho}[(2+\alpha(\theta, \rho)) u, u] d \rho \\
& \quad-c_{2} \int_{0}^{h} \frac{\phi^{2}}{\rho^{2}}\|u\|^{2} d \rho-c_{3} \int_{0}^{h} \phi^{2}\|u\|^{2} d \rho \\
& \quad-c_{4} \int_{0}^{h}\left|\phi \phi^{\prime}\right|\|u\|^{2} d \rho-\frac{c_{1}}{h} \exp \left(2 n h^{-\delta}\right) .
\end{aligned}
$$

Since $\alpha(\theta, \rho) \rightarrow 0$ uniformly in $\theta$ as $\rho \rightarrow 0$, we see

$$
\begin{aligned}
& \int_{0}^{h} \frac{n \delta(\delta+1)}{\rho^{\delta+2}} \phi^{2}\|u\|^{2} d \rho-\int_{0}^{h} \frac{\left|\phi \phi^{\prime}\right|}{\rho}[(2+\alpha(\theta, \rho)) u, u] d \rho \\
& \geqq \int_{0}^{h} \frac{n \delta}{\rho^{\delta+2}}(\delta-1-\alpha(\rho)) \phi^{2}\|u\|^{2} d \rho,
\end{aligned}
$$

where $\alpha(\rho) \rightarrow 0$ as $\rho \rightarrow 0$. Hence we can take $\delta$ as an arbitrary number larger than 1 , if $h$ is taken sufficiently small.

Remark 1. The decomposition of (4.12) is due to Mizohata [10].

Remark 2. I. S. Bernstein [2] proved the uniqueness in Cauchy problem for the elliptic operator with distinct characteristic roots without dimensional condition. He treated the problem by polar coordinate system $(r, \theta)$. We used the above coordinate system $(\rho, \theta)$ instead of the polar coordinate system.

5. First we prove Theorem 2. By an adequate coordinate transformation we can take $\Omega=\Omega_{a, d}$ and $\Gamma=\Gamma_{a, d}$. The exponent $\delta$ will be determined later. We have from Corollary 1

$$
\frac{\partial^{i+j} u}{\partial x^{i} \partial y^{j}}=o\left(\exp \left(-r^{-\delta+\varepsilon}\right)\right) \quad(r \rightarrow 0) \quad \text { in } \begin{aligned}
& \Omega_{a, d / 2} \\
& i+j \leqq m-1,
\end{aligned}
$$


where $\varepsilon$ can be taken as an arbitrary small positive number. By pro position 5 there is a positive constant $\boldsymbol{\delta}_{0}$ such that if $\boldsymbol{\delta}_{0}<\boldsymbol{\delta}-\boldsymbol{\varepsilon}$,

$$
\begin{aligned}
& \sum_{j=1}^{m} \int_{0}^{h} \phi_{n}(\rho)^{2}\left\|w_{j \rho}+\frac{1}{\rho}\left(Q_{j}+i P_{j}\right) w_{j \theta}\right\|^{2} d \rho \\
& \quad \geqq-\frac{c_{1}}{h} \exp \left(2 n h^{-\delta 0}\right)+c_{2} n \sum_{j=1}^{m} \int_{0}^{h} \frac{\phi_{n}(\rho)^{2}}{\rho^{\delta 0+2}}\left\|w_{j}\right\|^{2} d \rho
\end{aligned}
$$

where $\phi_{n}(\rho)=\exp \left(n \rho^{-\delta_{0}}\right)$. Hence we see from (3.7)

$$
\begin{array}{r}
\int_{0}^{h} \phi_{n}^{2}\|\mathscr{B} u\|^{2} d \rho+\frac{c_{1}}{h} \exp \left(2 n h^{-\delta 0}\right) \\
\geqq c_{3} n \sum_{j=1}^{m} \int_{0}^{h} \frac{\phi_{n}^{2}}{\rho^{\delta 0+2}}\left\|w_{j}\right\|^{2} d \rho .
\end{array}
$$

By Poincaré's inequality we have

$$
\|u\| \leqq \text { const. }\left\|u_{\theta}\right\| \text {. }
$$

On the other hand

Hence

$$
u_{\theta}=u_{x}\left(\rho \cos ^{2} \theta\right)_{\theta}+u_{y}(\rho \cos \theta \sin \theta)_{\theta} .
$$

$$
\|u\| \leqq \text { const. }\left(\left\|u_{x}\right\|+\left\|u_{y}\right\|\right)
$$

Using successively this inequality, we get

$$
\|\mathscr{B} u\| \leqq \operatorname{cost} . \sum_{j=1}^{n}\left\|w_{j}\right\| \text {. }
$$

Combining (5.1) and (5.2), we obtain

$$
c_{4}(h n)^{-1} \exp \left(2 n h^{-\varepsilon 0}\right) \geqq \sum_{j=1}^{m} \int_{0}^{h} \frac{\phi_{n}^{2}}{\rho^{\delta_{0}+2}}\left\|w_{j}\right\|^{2} d \rho
$$

Therefore when we take $\delta>\delta_{0}+\varepsilon$, we see that it is contradict if $\sum_{j=1}^{m}\left\|w_{j} \mid\right\|^{2} \neq 0$ in the neighbourhood of $\rho=0$. By classical procedure we see $w_{j} \equiv 0$ in $\Omega$. Thus the first assertion of Theorem 2 has been proved.

Next we consider the case where $m=2$ and the coefficients $a_{i j}$ of the principal part are real. Let us set $\delta=1+\varepsilon(\varepsilon>0)$. Then we have similarly

$$
\frac{\partial^{i+j} u}{\partial x^{i} \partial y^{j}}=o\left(\exp \left(-r^{-1-\varepsilon^{\prime}}\right)\right) \quad\left(r \rightarrow 0, \varepsilon^{\prime}>0\right) i+j \leqq 1
$$


We can assume $a_{i j}(0)=\delta_{i j}$ by a coordinate transformation. Thus we can apply Corollary 3 in place of Proposition 5, and we can follow the same process as in the proof of the first assertion. Hence we have finished the proof of Theorem 2. If the equation (1.3) is reduced to the equation (3.7), we see that $g=0$. Therefore the proof of Theorem 1 is contained in that of Theorem 2 as a special case.

\section{REFERENCES}

[1] N. Aronszajn, A unique continuation theorem for solutions of elliptic partial differential equations or inequalities of second order, J. Math. Pures Appl., 36 (1957), 235-249.

[2] I. S. Bernstein, On the unique continuation problem of elliptic partial differential equations, J. Math. \& Mech., 10 (1961), 579-606.

[3] A. P. Calderón, Uniqueness in the Cauchy problem for partial differential equations, Amer. J. Math., 80 (1958), 16-36.

[4] T. Carleman, Sur un problème d'unicité pour les systémes d'équations aux dériveés partiells à deux variables indépendants, Arkiv Mat., 26 B (1938), 1-9.

[5] E. Heinz, Übber die Eindeutigkeit beim Cauchyschen Anfangswertproblem einer elliptischen Differentialgleichung zweiter Ordnung, Nach. Akad. Wiss. Göttingen 1 (1955), 1-12.

[6] F. John, Continuous dependence on data for solutions of partial differential equations with a prescribed bound, Comm. Pure Appl. Math., 13 (1960), 551-585.

[7] E. M. Landis, On some properties of elliptic equations, Dokl. Akad. Nauk SSSR 107 (1956), 640-643 (Russian).

[8] M. M. Lavrentév, On Cauchy's boundary value problem for linear elliptic equations of the second order, Dokl. Akad. Nauk SSSR, 112 (1957) 195-197 (Russian).

[9] S. N. Mergelyan, Harmonic approximation and approximate solution of Cauchy's problem for Laplace equation, Dokl. Akad. Nauk SSSR, 107 (1956), 644-647 (Russian).

[10] S. Mizohata, Unicité du prolongement des solutions des équation elliptiques du quatrième ordre,.Proc. Japan Acad., 34 (1958), 687-692. 
